\title{
Self-writing a depressed index waveguide in bulk glass
}

\author{
Ami M. Ljungström and Tanya M. Monro \\ Optoelectronics Research Centre, University of Southampton, SO17 1BJ, UK
}

\begin{abstract}
We use numerical simulations to predict the first complex self-written structure in a bulk material. A depressed-index "pipe" structure, which guides light like a channel, is created using a LaguerreGaussian "donut" beam.
\end{abstract}

A self-written waveguide can dynamically evolve in response to illumination in a photosensitive material [1]. We recently reported self-written taper structures created in bulk chalcogenide glass [2], and waveguides have been written in bulk photopolymers [3,4]. Although in all these materials illumination increases the refractive index, some materials can exhibit a decrease (for example Ge-doped silica films [5]) and we recently observed such a decrease in bulk Nd-doped Bk7 glass. Negative index changes allow a new range of waveguide structures to be self-written, and this has not been previously explored.

We here report the first numerical simulations of self-writing a depressed index structure, and present simulations of complicated self-written structures in a bulk geometry. Note that few simulations have been carried out in the bulk geometry as these calculations are computationally intensive. The model used for self-writing is described in detail in Ref $[1,2,6]$. The paraxial wave equation describes the propagation of light, and a phenomenological photosensitivity equation describes the refractive index evolution. The equations are solved numerically using a split step beam propagation model.

Previous theoretical work shows that the shape of the self-written waveguide that evolves can be tailored via the writing beam [6]. If a material experiences a decrease in index in response to light, the diffraction of a simple Gaussian beam would increase. Therefore a more complicated writing beam is required to form a useful waveguide, and here an $\mathrm{m}^{\text {th }}$ order Laguerre-Gaussian "donut" beam is used [7]:

$$
E(x, y, t)=\sqrt{\frac{1}{\pi \mathrm{m} !}}\left(\frac{\sqrt{2}}{a}\right)^{\mathrm{m}+1} \exp \left(i \mathrm{~m} \tan ^{-1} \frac{y}{x}\right)\left(x^{2}+y^{2}\right)^{\frac{\mathrm{m}}{2}} \exp \left(-\frac{x^{2}+y^{2}}{a^{2}}\right)
$$

$\mathrm{E}(\mathrm{x}, \mathrm{y}, \mathrm{t})$ is the electric field amplitude, and $\mathrm{x}$ and $\mathrm{y}$ are the transverse coordinates. The beam width at the input face is $a$, corresponding to a peak-to-peak (PTP) value of the intensity distribution of $a \sqrt{2 m}$. In practice a "donut" shaped writing beam can be produced by several different techniques [8].

If this intensity distribution is used, the sample is illuminated by a ring of high intensity with a null in its centre. The refractive index decreases most where the intensity is highest, hence a ring-shaped depressed index structure starts to form at the input face. Over time the structure evolves and indeed our numerical simulations indicate that a relatively uniform unexposed region remains along the propagation axis. In Fig 1 a donut beam with $\mathrm{m}=1, \mathrm{PTP}=30 \mu \mathrm{m}$, and $\lambda=488 \mathrm{~nm}$ is used (the saturation index change is $-10^{-4}$ ). Our results show that the width of the unexposed central region can be controlled via $m$ and PTP.

The propagation characteristics of the induced depressed-index "pipe" structures have been determined by sending a Gaussian beam through the structure. Unsurprisingly, different guidance properties are obtained for structures written by donut beams of different widths. Fig 2 shows the intensity distribution of a Gaussian beam as it propagates through structures written using different beam widths. The cross-sections of the outcoming Gaussian beams are shown in the insets together with the same beam freely diffracting over this distance. It can clearly be seen how the central portion of beam is confined and guided by the structures.

We have presented simulations of a depressed refractive index self-written structure, this also represents the first simulations using a complex writing beam in a bulk geometry. This structure can be used to guide light. Here a first order Laguerre-Gaussian donut beam is considered, however higher order beams, and different PTP values, offer the flexibility to tailor the properties and characteristics of the final waveguide structure. 


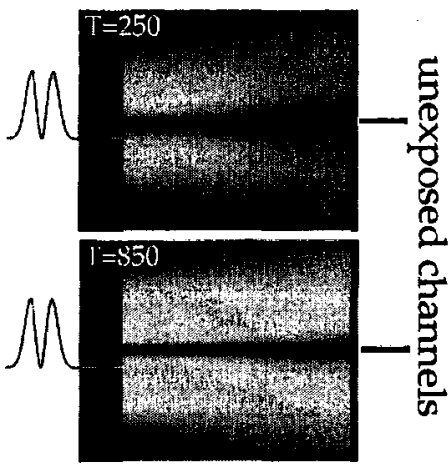

Fig. 1. Refractive index distribution along one transverse direction during the evolution of a depressed index structure, $\mathrm{PTP}=30 \mu \mathrm{m}$. A channel of relatively higher index evolves along the propagation axis.

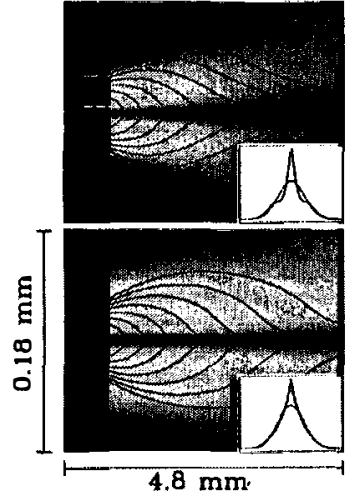

Fig. 2. A Gaussian beam (FWHM $=10 \mu \mathrm{m}, \lambda=1047 \mathrm{~nm}$ ) propagating through structures with PTP values of 20 (top) and $30 \mu \mathrm{m}$ (bottom). Insets show the outcoming guided beam together with beams travelling the same distance by free diffraction.

1. T.M. Monro, C. M. de Sterke, and L. Poladian, "Catching light in its own trap," J. Mod.Opt. 48, 191-238 (2001).

2. A. M. Ljungström, and T. M. Monro, "Light-induced self-writing effects in bulk chalcogenide glass," to appear in J. Lightwave Technol. 20, (2002).

3. S. Shoji, and S. Kawata, "Optically-induced growth of fiber patterns into a photopolymerizable resin," Appl. Phys. Lett. 75, 737-739 (1999).

4. M. Kagami, T. Yamashita, and H. Ito, "Light-induced self-written three-dimensional optical waveguide," Appl. Phys. Letț. 79, 1079-1081 (2001).

5. K. Simmons-Potter, and J. H. Simmons, "Band bleaching and growth dynamics in $45 \% \mathrm{GeO}_{2}-55 \% \mathrm{SiO}_{2}$ films," Appl. Phys Lett. 66, 2104-2106 (1995).

6. T. M. Monro, C. M. de Sterke, and L. A. Poladian, "Analysis of self-written waveguides in photopolymers and photosensitive materials," Phys. Rev. E 57, 1104-1113 (1998).

7. A. E. Siegman, Lasers (University Science Books, 1986), pp. 627-628.

8. N. R. Heckenberg, R. McDuf, C. P Smith, H. Rubinsztein-Dunlop, and M. J. Wegener, "Laser beams with phase singularities," Opt. quảnt. Electron., 24 , 951-962 (1992). 